\title{
CONSIDERAÇÕES EPISTEMOLÓGICAS SOBRE A INCORPORAÇÃO DO PRAGMATISMO CLÁSSICO NOS ESTUDOS DA INFORMAÇÃO
}

\author{
EPISTEMOLOGICAL CONSIDERATIONS ON THE \\ INCORPORATION OF CLASSICAL PRAGMATISM IN \\ THE INFORMATION STUDIES
}

\author{
Sônia Cristina Bocardi Moraesa \\ Carlos Cândido Almeida ${ }^{\mathrm{b}}$
}

\begin{abstract}
RESUMO
Introdução: A relação epistemológica contemplada pelo pragmatismo de Charles S. Peirce (1839-1914) deve ser observada a partir das incorporações do conceito original peirceano no campo da Ciência da Informação para evitar distorções no emprego do termo. O conceito de signo usado de uma maneira generalizada, assim como 0 pragmatismo, em suas versões posteriores a obra de Peirce geram uma diversidade de abordagens que não se adequam ao entendimento da concepção original. $O$ pragmatismo peirceano com abordagens que incluem a metodologia pragmaticista e a semiótica nos processos de organização do conhecimento devem ter sua contribuição revista na ciência da informação Objetivo: O objetivo deste trabalho é apontar as contribuições epistemológicas do pragmatismo peirceano à ciência da informação e, em particular, à organização da informação e do conhecimento. Metodologia: A análise teórica, visando à interpretação da organização do conhecimento tem, na pesquisa bibliográfica, fundamentação para relação entre a teoria pragmaticista peirceana e sua incorporação na ciência da informação. Resultados: Os processos de indexação são possibilidades de interpretação e não regras mentais de associação diádica entre significante e significado, entre conteúdo documental e um termo. A evolução de interpretantes e o encadeamento informacional criativo, presente na aquisição de conhecimento, estão contidos no método pragmático aplicável à organização do conhecimento usando sua característica de método científico. Conclusões: A aceitação das etapas criativas pragmáticas no ato de indexação implica em conceber esta característica epistemológica de criação que alia o conteúdo do documento à recuperação da informação.
\end{abstract}

Descritores: Informação. Pragmatismo Peirceano. Organização do Conhecimento.

\footnotetext{
a Docente do curso de Arquitetura e Urbanismo na UNIMAR Universidade de Marilia, Doutoranda em Ciencia da Informação, Mestre em Filosofia pela UNESP, Arquiteta e Urbanista -PUC Campinas. soniamoraesarquitetura@hotmail.com

b Departamento de Ciência da Informação, Faculdade de Filosofia e Ciências, Universidade Estadual Paulista - UNESP. carlosalmeida@marilia.unesp.br
} 
Interpretante.

\section{INTRODUÇÃO}

A análise teórica visando à organização da informação e do conhecimento é busca recorrente para a ciência da informação. Smiraglia (2002), descrevendo os esforços da área para entender a estrutura do conhecimento, verifica o uso de "teoria" enquanto conceito capaz de prever e gerenciar fenômenos. A capacidade explanatória está no alcance do uso da teoria. Smiraglia (2002, p. 331) considera que esta necessidade de quantificar os fenômenos a partir de observação controlada tem um paradigma positivista dominante, e este não é o aspecto principal para aquisição de conhecimento. O questionamento entre divisão de teoria e prática, pensamento e ação, mente e matéria norteia a busca processual para o entendimento das relações observadas cientificamente, e daria uma visão integrada desta dissociação positivista.

Para Hjorland (1998, p. 607, tradução nossa), teoria em ciência da informação é também definida como "uma explicação teórica da eficiência dos sistemas de informação, de comportamento do usuário, da função de diferentes elementos de pesquisa, como descrições, citações, títulos, e assim por diante". A noção de teoria desenvolvida utilizada tanto na ciência da informação quanto na organização do conhecimento tem relação direta com o método científico, o qual recebeu as contribuições do pragmatismo clássico.

A abordagem pragmatista deve instrumentalizar a busca da informação com análise e objetivos a serem contemplados. O pragmatismo, como método que leva em conta crenças e conceitos para Charles Peirce (1839-1914), determina a conduta com vistas ao objetivo a ser alcançado. Há sempre um movimento dos signos em direção a uma ação futura, e a semiótica, neste caso, é por ele considerada uma "lógica da conduta". Do ponto de vista definido neste artigo, a ação deve ser a de tornar cada vez mais clara a relação entre as considerações epistemológicas derivadas do pragmatismo e a organização da informação e do conhecimento, em especial, os métodos de indexação com vistas a recuperação em futuro das possibilidades de significação. 
Peirce contrapõe o pensamento moderno cartesiano e o conhecimento a partir da dúvida hipotética ao pensamento escolástico (PEIRCE, 1974, CP 5.264). Defende Peirce que não é possível abandonar as próprias crenças e duvidar delas automaticamente para chegar à verdade, como descrito no cartesianismo, pois aquilo que é observado desperta a admiração, necessidade de conhecimento, e a formulação de hipóteses parte dessa possibilidade de interação com o objeto, naquilo que é capaz de despertar a atenção do investigador. O pensamento é mediado por signos, e o conhecimento é um processo de investigação continuo, em busca da verdade que pode dar, mais adiante, o conhecimento esperado daquilo que é investigado (PEIRCE, 1974, CP 5.265). Nesse sentido, o método pragmático é a doutrina que trabalha a interação entre o signo e representação, de maneira a tornar conhecido aquilo que está na interação com o objeto, por meio deste processo.

Basta dizer mais uma vez que o pragmatismo não é, por si só, doutrina da metafísica, nem tentativa de determinar nenhuma verdade das coisas. É apenas um método para determinar os significados das palavras difíceis e dos conceitos abstratos. Todos os pragmáticos de qualquer espécie concordarão cordialmente com essa afirmação. Quanto aos efeitos ocultos e indiretos da prática do método pragmático, isso é outra questão. (PEIRCE, 1974, CP 5.464)

O conhecimento não é uma teoria dada a priori, a ser verificada na prática. No pragmatismo são considerados os efeitos práticos da relação de representação e a conduta por eles determinada, tendo a significação como decorrência deste processo. O significado é um tipo de antecipação em que se projeta no futuro as reações ao objeto considerado.

O pragmatismo peirceano com abordagens que incluem a metodologia pragmaticista e a semiótica nos processos de organização do conhecimento deve ter sua contribuição revista no âmbito da ciência da informação. O objetivo deste trabalho é apontar as contribuições epistemológicas do pragmatismo peirceano, à ciência da informação e, em particular, à organização da informação e do conhecimento. Esta comunicação relaciona-se com uma pesquisa doutoral em desenvolvimento que trata das contribuições para a Ciência da Informação do conceito de informação encontrado na filosofia peirceana. 


\section{PRAGMATISMO CLÁSSICO}

A contribuição de Charles Peirce, à filosofia principalmente, decorre, em princípio, do reconhecimento do seu contemporâneo e amigo, co-fundador do Clube Metafísico em 1872, o filósofo e psicólogo William James (1842-1910), cujos estudos de pragmatismo vêm da concepção peirceana, apesar de diferenças fundamentais. Os trabalhos desses pioneiros, bem como de John Dewey (1859-1952), inauguraram o pragmatismo clássico, também chamado simplesmente de pragmatismo norte-americano. Nesta seção são expostos alguns conceitos básicos associados ao pragmatismo clássico, especialmente 0 de extração peirceana.

Os artigos de Peirce "A fixação das crenças", de 1877 (PEIRCE, 1974, CP 5.358) 1 e "Como tornar nossas ideias claras", de 1877 (PEIRCE, 1974, CP 5.388) são parte de um conjunto de seis artigos publicados no periódico Popular Science Monthly, e são considerados como o começo dos estudos pragmatistas, mais tarde chamados por Peirce "Pragmaticismo" (HAUSSMAN, 1997, p. 20). Nestes trabalhos encontram-se que as quatro condições para inquirição do pensamento lógico, que são: 1) passar de um estado de incerteza para crença, 2) chegar a uma conclusão verdadeira, 3) fazendo uso do método científico e 4) que parte de uma hipótese que justifique esta busca pela verdade. $O$ estado de incerteza, ou dúvida, não é o mesmo como se apresenta no cartesianismo, mas uma possibilidade de levar adiante um estado de crença que pode ser testado e confrontado com a realidade em uma condição de dúvida verdadeira, a partir do hábito mental e de comportamento preexistente (HAUSSMAN, 1997, p. 27).

A formulação da hipótese tem uma sequência lógica de argumentação desenvolvida a partir da classificação dos signos, efetuada detalhadamente dentro da estrutura de classificação das ciências concebida por Peirce (PEIRCE, 1974, CP 1.176). Nesta classificação, a filosofia é concebida enquanto ciência

\footnotetext{
${ }^{1}$ Collected Papers é uma publicação póstuma dos escritos de Charles S. Peirce. Estão reunidos nela manuscritos inéditos e publicações feitas em vida. Para a citação desta obra estudiosos do autor fazem a referência conforme é usada nesta publicação, onde CP indica Collected Papers, o número seguinte indica o volume, e depois do ponto, tem-se a indicação do parágrafo. Desta forma, é citado o parágrafo numerado para apresentar a exata referência, tanto no livro impresso quanto na versão digital.
} 
do fato, vinda da observação da natureza; é a partir da condução das ações que os processos de significação podem ser percebidos (PEIRCE, 1974, CP 1.251), e então das informações obtidas no processo de significação, chegar à representação e à generalização. A tendência da repetição de determinadas condutas que podem ser representadas em um conceito, caracteriza um hábito (PEIRCE, 1974, CP 6.21). A experiência, quando tem sua concepção generalizada, proporciona, ao hábito, a ação de maneira facilitada, informando um arcabouço daquilo que pode ser realizado sem grandes esforços.

A inquietação causada pela formulação de uma hipótese, que pode alterar o hábito existente, move o sujeito cognoscente em direção à conclusão da hipótese aventada enquanto inquirição ou pesquisa científica, em busca da comprovação da verdade pelo método científico. Formular uma hipótese é disponibilizar elementos para essa comprovação lógica e a mediação da ação e representação é feita pelos signos.

Os termos gerais são signos, tidos enquanto representação que possibilita a articulação das hipóteses, seja em termos linguísticos ou diagramas matemáticos.

Um signo, ou representamen, é alguma coisa que representa alguém para algo em algum aspecto ou capacidade. Trata de alguém, isto é, cria na mente daquela pessoa um signo equivalente, ou talvez um signo mais desenvolvido. Aquele signo que ele cria chama o interpretante do primeiro signo. $O$ signo representa algo, seu objeto. Ele representa esse objeto, não em todos os aspectos, mas em referência a um tipo de ideia, que às vezes chamei de base do representamen (PEIRCE, 1974, CP 2.228).

Apesar de a tradição filosófica e a linguística se valerem do conceito de signo para entender o significado (meaning), no pragmatismo peirceano 0 significado de conceitos gerais ou termos são compreendidos como disposições, hábitos, leis que têm formulação também em expressões linguísticas (HAUSSMAN, 1997, p. 57). Estes são estendidos aos processos naturais nos quais a informação também está presente, independentemente da participação, ou mesmo da compreensão humana.

O estabelecimento de como devem ser todos os signos, para uma inteligência capaz de aprender com a experiência, é o objetivo da semiótica 
(SILVEIRA, 2007, p. 38). A divisão em classes de signos ou a maneira possível desses relacionamentos são as várias possibilidades de estruturação entre os entes signo, objeto e interpretante.

$O$ trabalho do pensamento peirceano, na tessitura de suas descobertas cientificas e filosóficas amplifica sua arquitetura metafísica. A semiótica, dentro das ciências normativas descreve as relações entre os signos num processo no qual a lógica está aliada a ética e estética. Portanto, uma teoria deve relacionarse com a capacidade de se admirar pelo novo (estética) e ter o compromisso e responsabilidade na condução para a busca da verdade (ética). Neste procedimento evolucionário, segue-se elencando meios lógicos através da ética para tornar esta significação verdadeira, alcançando o bem estético.

A estreita relação entre a significação, a determinação de conduta e aquilo que é objetivado, move a ação num processo sempre passível de adequação e correção da conduta para esta adequação. O bem estético almejado elenca técnicas e procedimentos norteados eticamente em direção ao objeto do signo. A máxima pragmática define esta relação entre a generalização e a apreensão do objeto oferecida durante o processo. "Considere quais efeitos que poderiam ter orientações praticas, nós concebemos o objeto que nossa concepção tem. Então, nossa concepção desses efeitos é a totalidade de nossa concepção do objeto[...]". (PEIRCE,1974, CP 5.2)

Assim, as hipóteses são confrontadas com a existência daquilo que é realizado no decorrer do processo com possibilidades de adequação e correção da conduta frente a expectativa de comprovação da hipótese aventada. A formulação de um significado racional, expressada na reunião das possíveis condutas tem, na concepção deste conjunto de conceitos, o significado obtido pelo método pragmático (PEIRCE, 1974, CP 5.412). A possibilidade, dentre todas as qualidades presentes na categoria fenomenológica primeiridade (contempla a qualidade de sentimento) tem, na factualidade da secundidade (categoria que compreende ação e reação), a execução da ação, e por conseguinte, a generalização e a representação na terceiridade (categoria da mediação).

A realidade é confrontada mediante os processos que envolvem as 
categorias fenomenológicas primeiridade, secundidade e terceiridade, concretizadas em pensamentos na forma de signos estão ativos e dinâmicos. $O$ significado está atrelado à geração de novos signos, isto é, os interpretantes ou efeitos apropriados de significação obtidos pela relação anterior dos signos (PEIRCE, 1974, CP 5.475). As três categorias perpassam todos os processos sígnicos na medida em que são fundamentais para a concepção dos signos. A partir da percepção e da ação fenomênica é possível alcançar a representação e o pensamento em terceiridade.

A relação entre signo e seu objeto, vistos enquanto aplicação ao pensamento humano tem, na continuidade, a grande contribuição peirceana. A continuidade evolucionária não está apenas no encadeamento temporal da formação de novos interpretantes a partir do anterior. O signo, a partir das qualidades emanadas do objeto, é capaz de generalizar este aspecto admirável percebido e ensejar uma representação, uma ideia gestada a partir da existência deste objeto. Assim se dá o signo, tanto parte do objeto representado quanto parte desta mente que selecionou os aspectos passíveis de aceitação para a representação realizada.

O idealismo objetivo (PEIRCE, 1974, CP 6.24) é considerado então uma espécie de monismo que junta mente e matéria nesta transposição do signo que informa, ao interpretante, quais os aspectos relevantes do objeto para sua realização em uma representação. Mente e matéria teriam uma mesma condição cognitiva, isto é, a matéria se configuraria como mente amalgamada e sedimentada em razão do tempo de evolução considerado (uma rocha, por exemplo) e mente como concebemos teria a mesma capacidade de produzir e romper com hábitos que a matéria, contudo, possuindo mais flacidez e agilidade nesse processo.

Signo, objeto e interpretante em uma relação genuína, evolucionária, fazem da relação signo-objeto, não uma representação diádica, de estar no lugar do outro apenas, mas supõe o envolvimento e o surgimento de um terceiro. $O$ interpretante nesta relação é capaz de apresentar um outro signo. A secundidade que apresenta a factualidade e o confronto com a existência proporciona o surgimento e a evolução do interpretante. Este último não pode se apresentar na 
forma degenerada (não triádica) em uma relação diádica apenas, concebida como um significante correspondendo ao significado.

A concepção de informação peirceana está contida na transmissão do objeto à formação de seu interpretante feita pelo signo (PEIRCE, 1974, CP 2.231). O processo de mediação entre a qualidade destacada do objeto em primeiridade, confrontada na ação na secundidade, na transmissão deste aspecto admirável, pode então ser generalizada numa representação em terceiridade, a qual forma o interpretante (PEIRCE, 1974, CP 1.537). Este interpretante, sendo ele mesmo um signo está sujeito a nova interação com outros aspectos a serem generalizados que alteram ou confirmam a representação anterior. É importante destacar a formação do interpretante que ocorre na mente humana e em outros fenômenos presentes na natureza, ambos tipos de formação supõem aprender com a experiência.

A evolução do processo de significação, de toda mente capaz de aprender com a experiência, fundamenta-se na definição de sinequismo (PEIRCE, 1974, CP 6.169) ou doutrina da continuidade. Entender o fenômeno pela articulação das hipóteses, mediados pelos signos é o transcurso possível da continuidade no pensamento e na natureza, considerando possível nova abordagem nos casos em que o processo resulte falível, uma vez que a continuidade "é a generalidade perfeita de uma lei de relacionamento" realizado (PEIRCE, 1974, CP 6.172).

A orientação prática das ações vai além de uma estratégia para obter um resultado, uma vez que o bem estético num objetivo a ser alcançado não depende de um desejo, mas de um propósito de conduta. A conduta dialógica entre o signo, aquilo que é representado e a condução lógica do processo sempre em correção para a melhor adequação é constante no processo.

No entanto, esta relação epistemológica do pragmatismo peirceano nem sempre é observada pelas incorporações conceituais no campo da ciência da informação, o que pode provocar distorções e deturpações. A substituição do objeto pelo signo que o representa traz a semiótica mais presente às questões de organização da informação e do conhecimento, porém a compreensão do método pragmático certamente tem contribuições de maior alcance para as 
pesquisas dentro da ciência da informação.

\section{PRAGMATISMO NA ORGANIZAÇÃO DA INFORMAÇÃO E DO CONHECIMENTO}

O que aqui se pretende, é realizar uma reflexão a respeito das contribuições do pragmatismo à área de organização do da informação e conhecimento dentro dos estudos da informação. Entende-se que a área de organização da informação volta-se para a representação de unidades de informação para posterior recuperação, e a organização do conhecimento trata das representações esquemáticas do conhecimento de um dado campo ou domínio. Tanto os processos de representação da informação como os processos de representação das estruturas de conhecimento não podem ser analisados isoladamente, por esta razão a expressão organização da informação e organização do conhecimento é utilizada em conjunto. No que tange à organização do conhecimento, as investigações em análise de domínio de Hjorland e Albrechtsen (1995, tradução nossa) já procuram a delimitação de um campo de atuação para recuperação de informação que possa incluir processos de busca automatizados. Thellefsen (2004, tradução nossa) faz um paralelo entre sua abordagem da teoria pragmaticista peirceana e uma metodologia que relacione a análise de domínio e a recuperação da informação. A organização do conhecimento é matéria de estudo recorrente, "[...] é um dos muitos campos que tentam desempenhar um papel nos ambientes futuros de comunicação e troca de conhecimento" (HJORLAND, 2008, p. 99, tradução nossa).

Especificamente, Hjorland relaciona os métodos de classificação a quatro abordagens filosóficas: empirismo, derivado da observação e da experiência; racionalismo, derivado do emprego da razão; historicismo, derivado da hermenêutica cultural; e o pragmatismo, relacionado à consideração de objetivos e suas consequências (HJORLAND, 1998, p. 612, tradução nossa).

Segundo o autor, dois problemas principais estão presentes na área: a independência entre a classificação e a cientificidade desta classificação e o método usado para isto, ou seja, este questionamento está presente quando a 
epistemologia é tratada na área. O empirismo está relacionado ao acoplamento bibliográfico, racionalismo em estruturação de indicações para o acesso, o historicismo na evolução temporal de assuntos e o pragmatismo associa-se aos "sistemas baseados em análise crítica do desenvolvimento e estado do conhecimento" (HJORLAND, 1998, p. 613, tradução nossa). Contudo, o conceito de pragmatismo adotado pelo autor não reflete o entendimento do pragmatismo peirceano, talvez nem mesmo o pragmatismo clássico; não há referência ao caráter metodológico deste, o que seria de grande importância para a ciência da informação tendo em vista os propósitos aplicados desta disciplina. Aqui não se trata da aplicação prática de um método pragmático, mas na compreensão de sua extensão a processos informacionais da ciência da informação.

No contexto da organização da informação, também se deve destacar a falta de um consenso das regras de indexação, e isto leva ao conteúdo semiótico de evolução sígnica, dentro do pragmatismo peirceano, para explicar a indexação. A representação, que apenas indica o conteúdo dos documentos, não tem a versatilidade dos algoritmos buscadores na Web para propor informação aos usuários

O processo semiótico da indexação foi largamente estudado (MAI, 1997, 2000, 2001; ALMEIDA; FUJITA; REIS, 2013). Contudo, a representação enquanto uma referência à realidade que não possibilita nova cadeia sígnica ou novo processo de significação, deixa a compreensão da teoria peirceana mais próxima de uma metodologia a ser reconhecida pela ciência da informação. Desta forma, a articulação de um método semiótico à indexação traz possibilidades de aplicação aos indexadores. Expandir o alcance da informação tem formas que vão além da eficiência para recuperação de conteúdo.

A proposta de estabelecer o perfil de conhecimento para determinar o signo fundamental, isto é, o cerne da representação ou caráter básico para a formação de um domínio do conhecimento (THELLEFSEN, 2004, tradução nossa), traça um paralelo entre a delimitação de um campo de atuação e um signo fundamental. Esta teoria, calcada no pragmatismo e na semiótica peirceana, intenta avaliar e produzir a representação visando à recuperação da informação, para tanto, envolve etapas e procedimentos. 
Neste aspecto, o signo fundamental deve ter repercussão naquela informação que é a busca do usuário. O tratamento do conteúdo da informação, particularmente a indexação, é um dos processos fundamentais no interior da organização da informação e do conhecimento (DAL'EVEDOVE; ALMEIDA; FUJITA, 2013). Segundo aponta os autores, "O ato de representar o conteúdo do documento com fins de recuperação futura nos sistemas de informação e documentação é o que torna a indexação uma atividade complexa e de contínua reparação." (DAL'EVEDOVE; ALMEIDA; FUJITA, 2013, p. 198). O signo fundamental é o conceito oferecido para que procedimentos de significação futuros de termos ocorram de maneira consistente.

Dada a sequência do processo informacional, a recuperação da informação requer incorporar a noção de hábito do pragmatismo peirceano. $O$ hábito regula a conduta enquanto um modo simplificador e organizador da atuação, pois os processos advindos da terceiridade têm seus significados reunidos na estruturação de leis (PEIRCE, 1974, CP 7.515). Aquilo que um hábito regula tem um arcabouço representacional já conhecido, facilitando a ação e a representação do processo no futuro.

A representação tem sua fundamentação teórica deixada em segundo plano na ciência da informação (EVANGELISTA; GUIMARÃES; ALMEIDA, 2014, p. 426). O comprometimento entre a representação do conteúdo e a organização da informação remete sobremaneira às concepções de hábito. Além disso, a ênfase da organização do conhecimento deve estar na proposta metodológica pragmaticista. Ambas, organização da informação e organização do conhecimento, devem voltar-se às ciências normativas, que aliam estética, ética e semiótica, bem como, reconsiderar a importância do conceito peirceano de verdade (uma relação genuína para a obtenção de informação) como suporte à noção de representação.

Os processos interpretativos tanto, do indexador quanto por parte daqueles que recuperam a informação, são passíveis de compreensão pela semiose ou de maneira redundante, semiose ilimitada (REIS, 2011). Os processos de indexação sendo possibilidades interpretantes e não regras mentais de associação do significante com o significado, estão abertos a novos 
processos de interação sígnica que darão origem a novos conhecimentos a partir da informação contida no assunto do documento, de maneira geral ou apenas naquele aspecto que for de interesse do usuário.

A probabilidade de sucesso na recuperação da informação está na capacidade de gerar interpretantes futuros. A classificação dos signos, vulgarmente utilizada, consiste tão somente no processo de dar nomes às etapas de indexação. O mais importante não é a caracterização dos signos, mas determinar condições para a ocorrência da continuidade na formação de novos interpretantes. A representação que tem a referência esgotada numa relação dual, que restringe as possibilidades de despertar interpretantes imediatos que sigam na cadeia de significação, característica do processo, voltem-se ao objeto, é uma informação limitada ou degenerada. A isto Peirce chama de "signo degenerado" (PEIRCE, 1974, CP 2.283), signo cuja informação determina seu interpretante de forma unívoca, sem expansão daquela representação. Mas a questão da noção de informação genuína e degenerada em Peirce, é assunto para outro trabalho.

\section{CONSIDERAÇÕES FINAIS}

A indexação possibilita a indicação do conteúdo com maiores ou menores probabilidades de recuperação dependendo inclusive do indexador. A representação para fins de documentação não deve ser uma referência que limita a cadeia sígnica, portanto, a evolução do conhecimento. Cabe ao indexador entender $o$ alcance do processo de representação, e isso 0 pragmatismo peirceano pode proporcionar.

Segundo Peirce, a significação apresenta-se como uma "[...] relação de qualidade ao assunto em que essa qualidade é inerente ao próprio assunto" (PEIRCE, 1974, CP 1.527). Em outras palavras, a representação do assunto se confunde com o próprio objeto e interrompe a relação sígnica triádica entre signo, objeto e interpretante. $O$ assunto representado no contexto da indexação não segue o processo que por natureza deve ser evolucionário, pois há um cerceamento da espontaneidade em razão da organização e da recuperação dos 
conteúdos. Contudo, é o retorno à primeiridade, ou seja, a possibilidade de abertura a novo processo de significação que leva aquele encadeamento a um novo signo. O propósito, o fim na busca de um objetivo ou a significação em se tratando de informação, é aquilo que faz da relação semiótica (representacional) um processo evolucionário. Não obstante, será no teste a ocasião da verificação da verdade obtida a cada nova hipótese de representação.

A semiose dentro do processo de indexação, tal como estudado (MAI, 1997, 2000, 2001; ALMEIDA; FUJITA; REIS, 2013), proporciona um novo passo no caminho da formação dos indexadores, apropriando-se da diferenciação entre a abertura para novas possibilidades de significação e a simples representação de demonstração de conteúdo nos processos de indexação.

Por fim, há um processo ético na representação do assunto e consiste na antecipação e na determinação da conduta de cada usuário na busca por informação. Esse processo vai proporcionar uma nova semiose, em outros níveis além da organização da informação. Este próximo passo de investigação da conduta de indexadores requer o entendimento do pragmatismo enquanto busca pela informação correta e ajustada ao objeto, enquanto processo contínuo que procura cientificamente a mais adequada e consistente representação, em outras palavras, a verdade.

\section{REFERÊNCIAS}

ALMEIDA, C. C. de; FUJITA, M. S. L.; REIS, D. M. dos. Peircean semiotics and subject indexing: contributions of speculative grammar and pure logic.

Knowledge Organization, v. 40, n. 4, p. 225-241, 2013. Disponível em: http://hdl.handle.net/11449/76267. Acesso em: 10 mai. 2017

DAL'EVEDOVE, P. R.; ALMEIDA, C. C. de; FUJITA, M. S. L. A interpretação de conceitos no processo de indexação: aspectos e reflexões semióticas. In: CONGRESSO ISKO ESPANHA E PORTUGAL, 1.; CONGRESSO ISKO ESPANHA, 11., 2013, Porto. Anais [...], Porto: Faculdade de Letras da Universidade do Porto; CETAC.MEDIA; ISKO, 2013. p. 190 - 203. Disponível em: http://www.iskoiberico.org/wp-content/uploads/2014/09/porto13.pdf. Acesso em: 10 mai. 2017

EVANGELISTA, I. V.; GUIMARÃES, J. A. C.; ALMEIDA, C. C. A semiótica como subsídio para a representação do conhecimento: uma análise conceitual 
sobre o tema. In: ENCONTRO NACIONAL DE PESQUISA EM CIÊNCIA DA INFORMAÇÃO, 15, 2014, Belo Horizonte. Anais [...], Belo Horizonte: ECI/UFMG, 2014. p. 413 - 429. Disponível em:

http://enancib2014.eci.ufmg.br/documentos/anais/anais-gt2. Acesso em : 10 mai. 2017

HAUSMAN, C. R. Charles S. Peirce's evolutionary philosophy. Cambridge: Cambridge University Press, 1997.

HJØRLAND, B. Theory and metatheory of information science: a new interpretation. Journal of Documentation, v. 54, n. 5, p. 606-621, 1998. Disponível em: https://www.emeraldinsight.com/doi/pdfplus/10.1108/EUM0000000007183. Acesso em: 05 mar.2019

HJØRLAND, B. What is Knowledge Organization (KO)? Knowledge Organization, v. 35, n. 2/3, p. 86-101, 2008. Disponível em: https://www.nomos-elibrary.de/10.5771/0943-7444-2008-2-3-86/what-isknowledge-organization-ko-jahrgang-35-2008-heft-2-3. Acesso em: 10 out. 2016

HJØRLAND, B.; ALBRECHTSEN, H. Toward a new horizon in information science: Domain-analysis. Journal of the American Society for Information Science, v. 46, n. 6, p. 400-425, jul. 1995. Disponível em: https://asistdl.onlinelibrary.wiley.com/doi/abs/10.1002/\%28SICI\%2910974571\%28199507\%2946\%3A6\%3C400\%3A\%3AAID-ASI2\%3E3.0.CO\%3B2-Y. Acesso em: 10 jun. 2020.

MAI, J-E. The Concept of Subject in a Semiotic Light. Proceedings of the ASIS Annual Meeting, Medford, v. 34, p. 54-64, 1997. Disponível em: http://jenserikmai.info/Papers/1997_TheConceptOfSubjectInASemioticLight.pdf. Acesso em: 10 mai. 2017

MAI, J-E. The Subject Indexing Process: an investigation of problems in knowledge representation. 2000. 345 f. Tese (Doutorado em Filosofia) Graduate School of Library and Information Science, The University of Texas, Austin, 2000. Disponível em: https://www.researchgate.net/profile/JensErik_Mai/publication/35253559_The_subject_indexing_process_microform_an_ investigation_of_problems_in_knowledge_representation/links/0 deec51ea5f75e 3ed5000000/The-subject-indexing-process-microform-an-investigation-ofproblems-in-knowledge-representation.pdf. Acesso em: 10 mai. 2017

MAI, J-E. Semiotics and indexing: an analysis of the subject indexing process. Journal of Documentation, v. 57, n. 5, p. 591-622, set. 2001. Disponível em: https://www.researchgate.net/profile/Jens-

Erik_Mai/publication/237537044_Semiotics_and_indexing_An_analysis_of_the subject_indexing_process/links/00b7d51ea64080a036000000/Semiotics-and- 
indexing-An-analysis-of-the-subject-indexing-process.pdf. Acesso em: 10 mai. 2017

PEIRCE, C. S. The Collected Papers of Charles Sanders Pierce. Cambridge: Harvard University Press, 1974. 1-8 v.

REIS, D. M. dos. Semiose ilimitada: contribuições para pensar na indexação de assunto. Revista EDICIC, v. 1, n. 4, p. 311-326, out./dez. 2011. Disponível em: https://dialnet.unirioja.es/descarga/articulo/3867153.pdf. Acesso em: 12 nov. 2018.

SILVEIRA, L. F. B. da. Curso de semiótica geral. São Paulo: Quartier Latin, 2007.

SMIRAGLIA, R. P. The Progress of Theory in Knowledge Organization. Library Trends, Urbana, v. 50, n. 3, p. 330-349, 2002. Disponível em: https://www.ideals.illinois.edu/bitstream/handle/2142/8414/librarytrendsv50i3d_ opt.pdf?sequence=1. Acesso em: 27 set. 2017

THELLEFSEN, T. Knowledge Profiling: The Basis for Knowledge Organization. Library Trends, Urbana, v. 52, n. 3, p. 507-514, 2004. Disponível em: https://www.ideals.illinois.edu/bitstream/handle/2142/1692/Thellefsen507514.pd f?sequence=2\&isAllowed=y. Acesso em: 10 jun. 2020.

\title{
EPISTEMOLOGICAL CONSIDERATIONS ON THE INCORPORATION OF CLASSICAL PRAGMATISM IN THE INFORMATION STUDIES
}

\begin{abstract}
Introduction: The epistemological relationship contemplated by the pragmatism of Charles S. Peirce (1839-1914) must observer the incorporations of the original Peircean concept in the field of Information Science to avoid distortions in the use of the term. The concept of sign used in a generalized way, as well as pragmatism, in its later versions, Peirce's work generates a diversity of approaches that do not fit the understanding of the original conception. Peircean pragmatism with approaches that include pragmatic and semiotic methodology in knowledge organization processes should have their contribution reviewed in information Science. Objective: The objective of this work is to point out the epistemological contributions of Peircean pragmatism to information science and, in particular, the organization of information and knowledge. Methodology: The theoretical analysis, aiming at the interpretation of knowledge organization has, in bibliographic research, a basis for the relationship between Peircean pragmatic theory and its incorporation in information science. Results: The indexing processes are possibilities of interpretation and not mental rules of dyadic association between signifier and signified, between documentary content and a term. The evolution of interpretants and the creative informational series, present in the acquisition of knowledge, are contained in the pragmatic method applicable to the organization of knowledge using its characteristic of scientific method. Conclusions: The acceptance of pragmatic creative
\end{abstract}


steps in the act of indexing implies conceiving this epistemological characteristic of creation that combines the content of the document with information retrieval.

Descriptors: Information. Peircean Pragmatism. Organization of Knowledge. Interpretant.

\title{
CONSIDERACIONES EPISTEMOLÓGICAS SOBRE LA INCORPORACIÓN DEL PRAGMATISMO CLÁSICO EN LOS ESTUDIOS DE INFORMACIÓN.
}

\begin{abstract}
RESUMEN
Introducción: La relación epistemológica contemplada por el pragmatismo de Charles S. Peirce (1839-1914) debe observarse a partir de las incorporaciones del concepto original de Peirce en el campo de la ciencia de la información para evitar distorsiones en el uso del término. El concepto de signo utilizado de forma generalizada, así como el pragmatismo, en sus versiones posteriores, el trabajo de Peirce genera una diversidad de enfoques que no se adaptan a la comprensión de la concepción original. El pragmatismo de Peirce con enfoques que incluyen metodología pragmática y semiótica en los procesos de organización del conocimiento debe tener su contribución revisada en la ciencia de la información. Objetivo: El objetivo de este trabajo es señalar las contribuciones epistemológicas del pragmatismo de Peirce a la ciencia de la información y, en particular, a la organización de la información y el conocimiento. Metodología: El análisis teórico, dirigido a la interpretación de la organización del conocimiento, tiene, en la investigación bibliográfica, una base para la relación entre la teoría pragmática de Peirce y su incorporación en la ciencia de la información. Resultados: Los procesos de indexación son posibilidades de interpretación y no reglas mentales de asociación diádica entre significante y significado, entre contenido documental y un término. La evolución de los interpretantes y la cadena creativa de información, presente en la adquisición de conocimiento, están contenidos en el método pragmático aplicable a la organización del conocimiento utilizando su característica del método científico. Conclusiones: La aceptación de pasos creativos pragmáticos en el acto de indexación implica concebir esta característica epistemológica de la creación que combina el contenido del documento con la recuperación de información.
\end{abstract}

Descriptores: Información. Pragmatismo Peirceano. Organización del Conocimiento. Interpretante.

Recebido em: 01.01.2020

Aceito em: 10.06.2020 